\title{
National survey on use of time-lapse imaging systems in IVF laboratories
}

\author{
Andrey V. Dolinko ${ }^{1,2}$ • L. V. Farland ${ }^{1,3}$ • D. J. Kaser $^{1}$ • S. A. Missmer ${ }^{1,3,4}$ • C. Racowsky ${ }^{1}$
}

Received: 9 March 2017 / Accepted: 22 May 2017 /Published online: 10 June 2017

(C) Springer Science+Business Media New York 2017

\begin{abstract}
Purpose Several time-lapse imaging (TLI) systems for noninvasive continuous monitoring of developing embryos are currently available. The present study explored the prevalence, means of acquisition, and clinical application of TLI systems in USA in vitro fertilization (IVF) laboratories.

Methods An online cross-sectional survey of 294 USA IVF laboratory directors was conducted in February and March 2016. Those directing more than one laboratory were asked to complete the survey for their home program and for
\end{abstract}

Andrey V. Dolinko

andolinko@gmail.com

L. V. Farland

lfarland@mail.harvard.edu

D. J. Kaser

dankaser@gmail.com

S. A. Missmer

stacey.missmer@channing.harvard.edu

C. Racowsky

cracowsky@partners.org

1 Department of Obstetrics, Gynecology, and Reproductive Biology, Division of Reproductive Endocrinology and Infertility, Brigham and Women's Hospital and Harvard Medical School, 75 Francis Street, ASB I-3, Boston, MA 02115, USA

2 Present address: Department of Obstetrics and Gynecology, Women \& Infants Hospital of Rhode Island, 101 Dudley Street, Providence, RI 02905, USA

3 Department of Epidemiology, Harvard T.H. Chan School of Public Health, 677 Huntington Ave, Boston, MA 02115, USA

4 Channing Division of Network Medicine, Department of Medicine, Brigham and Women's Hospital and Harvard Medical School, 181 Longwood Avenue, Boston, MA 02115, USA their smallest laboratory by number of IVF/intracytoplasmic sperm injection (ICSI) cycle starts. Use of TLI was analyzed using logistic regression to calculate odds ratios (OR).

Results Of 294 directors surveyed, 162 (55\%) reported data on 204 laboratories. Thirty-five laboratories (17\%) possessed at least one TLI system (median 2, interquartile range 1-4, total range 1-11). The more oocyte retrievals a laboratory performed annually, the more likely the laboratory was to possess a TLI system. Fifteen laboratories (43\%) purchased their own systems, while others leased, loaned, or received donated systems. Twenty-five laboratories (71\%) reported using TLI for embryo selection; all used TLI always, or usually, in combination with standard morphology evaluation. Twenty laboratories (80\%) offered TLI to all patients. Some laboratories charged patients for TLI. Directors with TLI systems were more inclined to believe that TLI has value for embryo selection in clinical IVF.

Conclusions TLI system possession in USA IVF laboratories is low, although positively associated with the number of retrievals performed and with directors' opinions on the technology's utility. Over 70\% of laboratories with TLI systems use them clinically, and less than half purchased their systems.

Keywords Assisted reproductive technology · Embryo selection · Morphokinetics $\cdot$ Time-lapse imaging

\section{Introduction}

A major challenge in in vitro fertilization (IVF) is to reduce the risk of multiple gestations. The ideal way to achieve this would be to perform exclusively single embryo transfer for every patient. This practice would be most effective if a technology was available enabling identification of the single most 
developmentally competent embryo in any given cohort. Time-lapse imaging (TLI) is one technology that has been actively investigated over the last several years as a means for adjunctive embryo selection. This technology allows for the non-invasive, continuous monitoring of developing embryos in an undisturbed culture environment. While over 20 morphokinetic markers have been identified [1], most of them have not been thoroughly validated in prospective or randomized controlled studies [2].

Several TLI systems are currently available for use in the clinical IVF laboratory. Based on the volume of research that has been published on TLI, it is clear that this technology is being actively pursued as a tool for embryo selection. Furthermore, an Internet search for TLI demonstrates that some infertility clinics are advertising their use of the technology in clinical situations. However, no data exist on how many clinics in the USA are currently using TLI and in what capacity.

The purpose of this study was to assess the current landscape of TLI availability, the means by which the systems were acquired, and the prevalence of clinical use for embryo selection in IVF laboratories across the USA. Answers to these questions will help set the stage for further evaluation of TLI use in clinical situations, uncover potential biases in implementation of the novel technology, and lay the groundwork for further study of the application of TLI in clinical IVF.

\section{Methods}

\section{Participants}

The Society for Assisted Reproductive Technology Clinical Outcome Reporting System (SART-CORS) database was used to compile a list of all IVF clinics in the USA. The database was subsequently queried to determine the name and e-mail address of the assisted reproductive technology (ART) laboratory director at each clinic. In instances where contact information was not available, the American Society for Reproductive Medicine (ASRM) database was cross-referenced and/or clinics were individually contacted by phone to obtain contact information for the laboratory director. Some directors were noted to direct several ART laboratories; these directors were asked to respond to the laboratory-specific questions on the survey twice-once for their home institution and once for their smallest laboratory according to the number of IVF/ICSI cycle starts annually.

\section{Survey design}

The survey was developed using a combination of binary, multiple-choice, and open-response questions. Some of the multiple-choice answers also provided respondents the opportunity to write-in their own answers. Prior to survey release, the laboratories were stratified into quintiles by size according to number of fresh retrievals performed in 2014, as reported in SART-CORS. A pilot survey was then sent to laboratory directors of one private and one university-affiliated laboratory from each of the five quintiles. As no survey items were identified as problematic by these pilot participants, no revisions were made to the survey.

\section{Survey distribution}

The survey was distributed in February-March 2016 to all laboratory directors whose e-mail addresses were available; up to three reminder e-mails were sent to those directors who had not responded within 5 weeks of survey release. Data acquisition was accomplished using Qualtrics (Qualtrics, March 2016, Provo, UT).

\section{Statistical analysis}

Data were analyzed using logistic regression to calculate odds ratios (OR) and 95\% confidence intervals (CI) and Wald twosided $p$ values according to the number of oocyte retrievals performed annually by a laboratory. Open-response data were compiled, and unifying themes were identified by a single author (AVD). The defined themes and included responses were reviewed and confirmed by the other authors. SAS statistical software version 9.3 was used for all analyses (SAS Institute Inc., Cary, NC).

\section{Results}

Of the 294 directors surveyed, 162 responded (55\%), with data available from 204 of 381 laboratories (54\%) in the USA (Table 1).

\section{Possession of TLI system}

The distribution of laboratories according to the possession of a TLI system is shown in Fig. 1. More than half of the 204 laboratories $(n=123,60 \%)$ did not have a system and had no plans to obtain one, 12 laboratories $(6 \%)$ had no system but had plans to get one, while 35 (17\%) had no system with their plans for acquisition being unknown (Fig. 1a). The remaining 35 laboratories (17\%) reported having at least one TLI system, with 6 of them $(17 \%)$ having two different types. The majority of those with only one type of system possessed either $\operatorname{EmbryoScope}^{\mathrm{TM}}(n=15,43 \%)$ or Eeva ${ }^{\mathrm{TM}}(n=10,29 \%)$ system(s), with the remainder having at least one Primo Vision $^{\mathrm{TM}}$, Miri® TL, or Astec Embryo Observation System CCM-IVF (Fig. 1b). Of those having at least one 
Table 1 Demographics of survey responders compared to all SARTCORS IVF clinics

\begin{tabular}{|c|c|c|}
\hline & Survey responders & $\begin{array}{l}\text { Number of } \\
\text { SART-CORS clinics }\end{array}$ \\
\hline \multicolumn{3}{|c|}{ No. of oocyte retrievals/year } \\
\hline $\begin{array}{l}\cdot \text { Up to } 99 \\
\cdot 100-299 \\
\cdot 300-499 \\
\cdot 500-999 \\
\cdot 1000-2000 \\
\cdot>2000\end{array}$ & $\begin{array}{l}28(13.7 \%) \\
79(38.7 \%) \\
40(19.6 \%) \\
3617.7 \%) \\
15(7.4 \%) \\
6(2.9 \%)\end{array}$ & $\begin{array}{l}87 \\
141 \\
62 \\
65 \\
28 \\
13\end{array}$ \\
\hline \multicolumn{3}{|l|}{ Clinic setting } \\
\hline $\begin{array}{l}\text { - Private } \\
\text { - University }\end{array}$ & $\begin{array}{l}155(76.0 \%) \\
49(24.0 \%)\end{array}$ & $\begin{array}{l}307(80.6 \%) \\
74(19.4 \%)\end{array}$ \\
\hline
\end{tabular}

EmbryoScope or Eeva system, 75.0\% (15/20) and 76.9\% (10/ 13), respectively, exclusively possessed one of these TLI systems.

Twenty-three of the 35 laboratories $(65.7 \%)$ were in private clinics, and $12(34.3 \%)$ were at university-affiliated programs. The median number of systems possessed by a laboratory was 2 (interquartile range $1-4$, total range $1-11$ ). Fifteen of the 35 laboratories $(42.9 \%)$ purchased their own systems, eight leased them (22.9\%), nine (25.7\%) had systems that were either loaned $(n=7,20.0 \%)$ or donated $(n=2,5.7 \%)$ by the manufacturer, one (2.9\%) had both purchased and loaned systems, and two (5.7\%) had systems of unknown acquisition.

Laboratories that performed more oocyte retrievals annually tended to have greater odds of possessing a TLI system (Fig. 2).

\section{TLI system use}

Of the 35 laboratories that possessed at least one TLI system, $25(71.4 \%)$ reported using TLI for selecting embryos for transfer. Seven (28.0\%) of the laboratories charged patients for use of a system, with charges ranging from $\$ 200$ to $>\$ 2000$ per cycle. All 25 laboratories reported using TLI in combination with standard morphology evaluation either always $(n=22$,
$88.0 \%$ ) or usually ( $n=3,12.0 \%)$; none used it alone. Two $(8.0 \%)$ laboratories used TLI for selection of cleavage-stage embryos for transfer (day 2 and/or day 3), nine (36.0\%) used TLI for selection of embryos to be transferred exclusively at the blastocyst stage (days 5 and/or 6), and 14 (56.0\%) used TLI for selection of embryos for transfer at both cleavage and blastocyst stages.

Twenty of the 25 laboratories ( $80.0 \%$ ) offered use of timelapse to all their patients. One of these laboratories indicated that they offered TLI to all of their patients, as well as to those who had previously failed an IVF cycle. Of the other five laboratories, one $(n=1,4.0 \%)$ gave preference to egg thaw cycles and PGD cases but otherwise randomly spaced cycles according to TLI system availability, while the remainder offered the technology based on availability and/or "lab director choosing" ( $n=1,4.0 \%)$, or only made the technology available to patients who were enrolled in a TLI clinical study $(n=2,8.0 \%)$, or who were "patients of interest" $(n=1,4.0 \%)$.

\section{Lab directors' opinions}

One hundred and fifty-seven of the 162 respondents provided opinions regarding the utility of TLI for embryo selection, of which $59(37.6 \%)$ believed that TLI is superior to standard morphology grading for the evaluation and selection of embryos. Thirty-four of the 156 (21.8\%) who reported having read the literature believed that published data support clinical use of TLI for selecting embryos for transfer. Furthermore, 34 of $153(22.2 \%)$ believed that TLI will become standard of care. Laboratory directors with TLI systems were significantly more likely to agree with each of the opinion statements above (see Table 2) than directors of laboratories without TLI systems ( $p<0.01$ for all statements).

Stratifying the opinions by the TLI system that laboratory directors possessed, those with an EmbryoScope tended to have more positive views of TLI than those with either the Eeva or only one of the other systems. Notably, about $70 \%$ of directors with an EmbryoScope believed that TLI is superior to standard morphology and that it would become the standard
Fig. 1 Distribution of laboratories based on TLI system possession

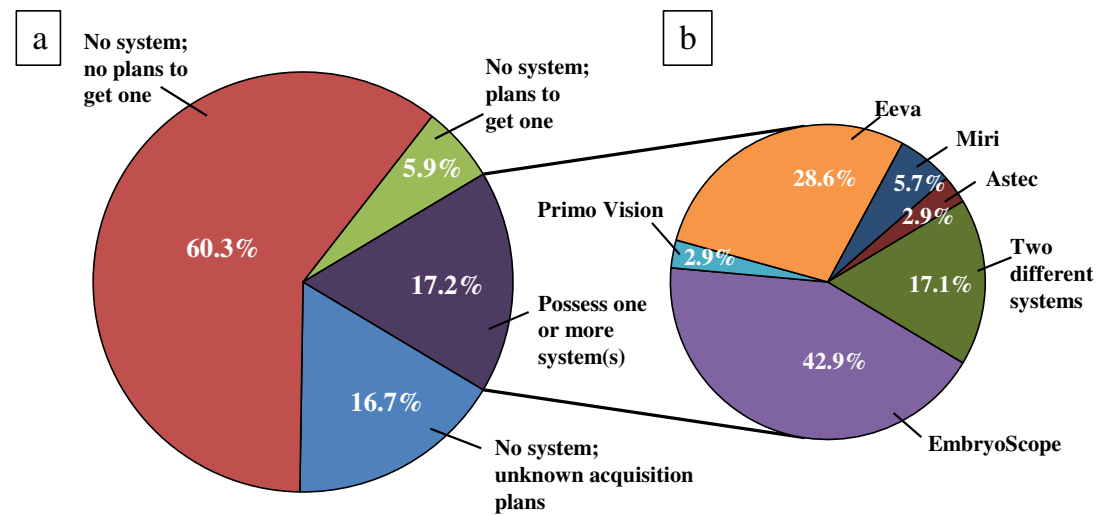




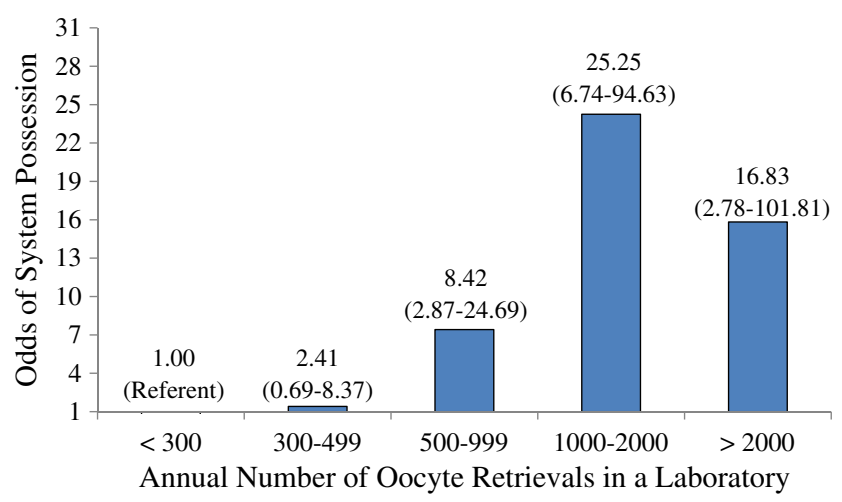

Fig. 2 Odds of TLI system possession based on clinic volume by annual number of oocyte retrievals. The odds ratios and $95 \%$ CIs are shown above each histogram; referent $=<300$ retrievals (no clinics with $<100$ retrievals possessed a TLI system)

of care for embryo selection, even though only just over half $(55 \%)$ believed that current literature supports clinical use of TLI.

In response to why laboratory directors think TLI will or will not become the standard of care, the following themes were noted. Statements in support of TLI cited undisturbed culture conditions ( $n=6)$, TLI used as a "tie breaker" for embryo selection $(n=4)$, and its use as an adjunctive tool that provides additional information to the embryologist $(n=3)$. Two lab directors noted that "it is superior to standard morphology evaluation" and "we have better results." Furthermore, several responses noted that TLI will become the standard of care if/when the cost decreases $(n=5)$, only in specific cases $(n=1)$, and only if studies demonstrate a benefit $(n=5)$. Specifically, directors with an EmbryoScope cited the following as reasons for why TLI will become the standard of care: (1) more data and efficiency, (2) improved outcomes, (3) future price decrease, and (4) undisturbed culture conditions.

Laboratory directors who think that TLI will not become the standard of care commented that the technology is too expensive $(n=35)$, that there is no evidence that it provides additional benefit $(n=22)$, and that it is too time-intensive and/or is impractical $(n=4)$. Some said that the only benefit of TLI is that the system provides an undisturbed culture $(n=2)$, that it will be beneficial only in specific cases $(n=2)$ or as a tie breaker $(n=1)$, that any potential benefit would depend on the quality of the laboratory itself $(n=6)$, and that more data are needed $(n=9)$. Furthermore, they noted that TLI analysis does not accurately predict ploidy status and/or that PGS is a more powerful tool $(n=26)$ and that it may be an appropriate tool for day 3 embryo transfers but not for day $5 / 6$ transfers $(n=7)$. Finally, two $(n=2)$ laboratory directors specifically cited poor performance of a certain TLI-S, stating that "embryologist observations gave as good or better pregnancy rates."

\section{Discussion}

In this study, we surveyed directors of IVF laboratories in the USA to assess the prevalence, means of acquisition, and clinical application of TLI systems in the USA. Based on the results of this survey, the prevalence of TLI systems in the USA is low (17.2\%) and more likely to be possessed by laboratories with greater numbers of oocyte retrieval cycles annually. The majority of laboratories with a TLI system(s) use it clinically, less than half purchased their system(s), and over one quarter charge patients for usage, albeit over a wide cost range. Of the 25 laboratories that use TLI clinically, all of them use it always or usually in combination with conventional morphology evaluation, and most offer TLI use to all of their patients. Those directors with a TLI system are more inclined to believe that such a system has value for embryo selection in clinical IVF.

In numerous retrospective studies, some morphokinetic markers, which are identifiable only with the use of TLI, have been shown to be predictive of blastocyst development [3-13], implantation [10, 14-16], or poor embryo potential $[5,7,17]$. Furthermore, use of TLI systems has the additional potential advantage of incubating embryos in an undisturbed culture environment. However, at this time, few RCTs have been performed to prospectively validate these markers or the overall use of TLI for embryo selection [18-21], and most have shown no improvement in clinical pregnancy or implantation rates (between 64 and 843 patients randomized). As several systematic reviews have concluded, existing evidence is currently insufficient to support the use of TLI for embryo selection $[2,22,23]$.

To our knowledge, this is the first study that has investigated the prevalence and use of TLI systems in clinical IVF in the USA.

Table 2 Directors' opinions on the value of TLI based on their laboratory's possession of a TLI system

\begin{tabular}{llll}
\hline Question to Directors & $\begin{array}{l}\text { Directors without TLI in } \\
\text { their lab(s) }(N=130)\end{array}$ & $\begin{array}{l}\text { Directors with TLI in } \\
\text { their lab(s) }(N=32)\end{array}$ & $\begin{array}{l}\text { Odds Ratio of Agreement } \\
(95 \% \mathrm{CI})\end{array}$ \\
\hline Is TLI superior to standard morphology? & $40 / 126^{\mathrm{a}}(31.7 \%)$ & $19 / 31(61.3 \%)$ & $3.40(1.51-7.69)$ \\
Does literature support clinical use of TLI? & $19 / 122(15.6 \%)$ & $15 / 31(48.4 \%)$ & $5.08(2.16-11.98)$ \\
Will TLI become standard of care? & $14 / 124(11.3 \%)$ & $20 / 29(69.0 \%)$ & $17.46(6.66-45.76)$ \\
\hline
\end{tabular}

${ }^{a}$ Number in agreement/Number of respondents 
The strengths of the study are that we attempted to survey the embryology laboratory directors from all IVF clinics in the USA; among them, more than $50 \%$ responded, and the survey responders were representative of SART-CORS clinics as reflected by a similar distribution of clinic setting (private vs. universityaffiliated) and clinic size by number of oocyte retrievals per year. In addition, the questionnaire created for this study was piloted to confirm clarity and validity. However, this study does have several limitations. First, to make the survey manageable for participants, we had to restrict responses to two clinics per director; this may have introduced bias with possible over-representation of some director's preferences and under-representation of others with respect to clinic possession and utilization of TLI systems. Furthermore, limiting the length of the survey prevented us from collecting additional potentially relevant data, including more demographic information on the laboratory directors, laboratory-specific culture conditions, and how laboratories changed their practices and/or outcomes with implementation of TLI systems. Also, this was a voluntary survey, so responses may not be representative of all clinics despite the observed distribution of clinic characteristics, and the questionnaire created for this survey study was not tested for reliability (confirming that the responders would have given the same answers if they completed the questionnaire more than once). Finally, these responses reflect the landscape of TLI utilization in the USA, and may not be representative of laboratories in other countries around the world.

In conclusion, while several TLI systems are FDA-approved and currently available commercially, we found that the percentage of laboratories that possess even one TLI system is low. Those laboratories that do have TLI tend to use the technology clinically, and about a quarter of those charge patients for the use of the technology. Further research should explore how TLI systems have changed clinical practice in the laboratories that have them, as well as reporting on how clinics are explaining the technology and its use to patients, particularly if they are using it only for certain populations. Furthermore, well-designed RCTs are needed to determine whether or not use of TLI significantly improves embryo selection and thus IVF success rates, and is of reasonable value and cost-effectiveness to implement into routine clinical practice.

Compliance with ethical standards This study was considered exempt by the Partners HealthCare Institutional Review Board.

Conflict of interest C.R. received financial support and the EEVA System and Test on loan from Progyny to perform a different study. The other authors declare that they have no conflict of interest.

\section{References}

1. Kaser DJ, Racowsky C. Clinical outcomes following selection of human preimplantation embryos with time-lapse monitoring: a systematic review. Hum Reprod Update. 2014;20(5):617-31. doi: 10.1093/humupd/dmu023.

2. Racowsky C, Kovacs P, Martins WP. A critical appraisal of timelapse imaging for embryo selection: where are we and where do we need to go? J Assist Reprod Genet. 2015;32(7):1025-30. doi:10. 1007/s10815-015-0510-6.

3. Wong CC, Loewke KE, Bossert NL, Behr B, De Jonge CJ, Baer TM, et al. Non-invasive imaging of human embryos before embryonic genome activation predicts development to the blastocyst stage. Nat Biotechnol. 2010;28(10):1115-21. doi:10.1038/nbt.1686.

4. Dal Canto M, Coticchio G, Mignini Renzini M, De Ponti E, Novara PV, Brambillasca F, et al. Cleavage kinetics analysis of human embryos predicts development to blastocyst and implantation. Reprod BioMed Online. 2012;25(5):474-80. doi:10.1016/j.rbmo. 2012.07.016.

5. Hashimoto S, Kato N, Saeki K, Morimoto Y. Selection of highpotential embryos by culture in poly(dimethylsiloxane) microwells and time-lapse imaging. Fertil Steril. 2012;97(2):332-7. doi:10. 1016/j.fertnstert.2011.11.042.

6. Cruz M, Garrido N, Herrero J, Perez-Cano I, Munoz M, Meseguer M. Timing of cell division in human cleavage-stage embryos is linked with blastocyst formation and quality. Reprod BioMed Online. 2012;25(4):371-81. doi:10.1016/j.rbmo.2012.06.017.

7. Desai N, Ploskonka S, Goodman L, Austin C, Goldberg J, Falcone T. Analysis of embryo morphokinetics, multinucleation and cleavage anomalies using continuous time-lapse monitoring in blastocyst transfer cycles. Reprod Biol Endocrinol. 2014;12(1):54.

8. Conaghan J, Chen AA, Willman SP, Ivani K, Chenette PE, Boostanfar R, et al. Improving embryo selection using a computer-automated time-lapse image analysis test plus day 3 morphology: results from a prospective multicenter trial. Fertil Steril. 2013;100(2):412-419 e5. doi:10.1016/j.fertnstert.2013.04.021.

9. Kirkegaard K, Kesmodel US, Hindkjaer JJ, Ingerslev HJ. Timelapse parameters as predictors of blastocyst development and pregnancy outcome in embryos from good prognosis patients: a prospective cohort study. Hum Reprod. 2013;28(10):2643-51. doi:10. 1093/humrep/det300.

10. Aparicio-Ruiz B, Basile N, Perez Albala S, Bronet F, Remohi J, Meseguer M. Automatic time-lapse instrument is superior to singlepoint morphology observation for selecting viable embryos: retrospective study in oocyte donation. Fertil Steril. 2016 Nov;106(6): 1379-1385.e10. doi: 10.1016/j.fertnstert.2016.07.1117.

11. Diamond MP, Suraj V, Behnke EJ, Yang X, Angle MJ, LambeSteinmiller JC, et al. Using the Eeva test adjunctively to traditional day 3 morphology is informative for consistent embryo assessment within a panel of embryologists with diverse experience. J Assist Reprod Genet. 2015;32(1):61-8. doi:10.1007/s10815-014-0366-1.

12. Petersen BM, Boel M, Montag M, Gardner DK. Development of a generally applicable morphokinetic algorithm capable of predicting the implantation potential of embryos transferred on day 3 . Hum Reprod. 2016;31(10):2231-44. doi:10.1093/humrep/dew188.

13. VerMilyea MD, Tan L, Anthony JT, Conaghan J, Ivani K, Gvakharia $\mathrm{M}$, et al. Computer-automated time-lapse analysis results correlate with embryo implantation and clinical pregnancy: a blinded, multi-centre study. Reprod BioMed Online. 2014;29(6): 729-36. doi:10.1016/j.rbmo.2014.09.005.

14. Meseguer M, Herrero J, Tejera A, Hilligsoe KM, Ramsing NB, Remohi J. The use of morphokinetics as a predictor of embryo implantation. Hum Reprod. 2011;26(10):2658-71. doi:10.1093/ humrep/der256.

15. Meseguer M, Rubio I, Cruz M, Basile N, Marcos J, Requena A. Embryo incubation and selection in a time-lapse monitoring system improves pregnancy outcome compared with a standard incubator: a retrospective cohort study. Fertil Steril. 2012;98(6):1481-1489 e10. doi:10.1016/j.fertnstert.2012.08.016. 
16. Liu Y, Chapple V, Feenan K, Roberts P, Matson P. Time-lapse deselection model for human day 3 in vitro fertilization embryos: the combination of qualitative and quantitative measures of embryo growth. Fertil Steril. 2016;105(3):656-662e1. doi:10.1016/j. fertnstert. 2015.11.003.

17. Cetinkaya M, Pirkevi C, Yelke H, Colakoglu YK, Atayurt Z, Kahraman S. Relative kinetic expressions defining cleavage synchronicity are better predictors of blastocyst formation and quality than absolute time points. J Assist Reprod Genet. 2015;32(1):2735. doi:10.1007/s10815-014-0341-x.

18. Goodman LR, Goldberg J, Falcone T, Austin C, Desai N. Does the addition of time-lapse morphokinetics in the selection of embryos for transfer improve pregnancy rates? A randomized controlled trial. Fertil Steril. 2016;105(2):275-285.e10. doi:10.1016/j.fertnstert. 2015.10.013.

19. Kahraman S, Çetinkaya M, Pirkevi C, Yelke H, Kumtepe Y. Comparison of blastocyst development and cycle outcome in patients with eSET using either conventional or time lapse incubators. A prospective study of good prognosis patients. J Reprod Biotechnol Fertil. 2012;3(2):55-61. doi:10.1177/205891581200300204.
20. Kaser DJ, Bormann CL, Missmer SA, Farland LV, Ginsburg ES, Racowsky C. Eeva ${ }^{\mathrm{TM}}$ pregnancy pilot study: a randomized controlled trial of single embryo transfer (SET) on day 3 or day 5 with or without time-lapse imaging (TLI) selection. Fertil Steril. 106(3): e312. doi: 10.1016/j.fertnstert.2016.07.886.

21. Rubio I, Galan A, Larreategui Z, Ayerdi F, Bellver J, Herrero J, et al. Clinical validation of embryo culture and selection by morphokinetic analysis: a randomized, controlled trial of the embryo scope. Fertil Steril. 2014;102(5):1287-1294.e5. doi:10.1016/j. fertnstert. 2014.07.738.

22. Armstrong S, Arroll N, Cree LM, Jordan V, Farquhar C. Time-lapse systems for embryo incubation and assessment in assisted reproduction. The Cochrane database of systematic reviews. 2015(2): Cd011320. doi:10.1002/14651858.CD011320.

23. Polanski LT, Coelho Neto MA, Nastri CO, Navarro PA, Ferriani RA, Raine-Fenning N, et al. Time-lapse embryo imaging for improving reproductive outcomes: systematic review and meta-analysis. Ultrasound Obstet Gynecol : Off J Int Soc Ultrasound Obstet Gynecol. 2014;44(4):394-401. doi:10.1002/uog.13428. 